\title{
Notiz uiber eine einfache Methode zur Darstellung von Tris(trimethylsilyl)phosphin
}

\author{
Gerd Becker* und Wolfgang Hölderich
}

Institut für Anorganische Chemie der Universität Karlsruhe, D-7500 Karlsruhe, Englerstr. 11

Eingegangen am 13. Januar 1975

Tris(trimethylsilyl)phosphin wird wegen seiner reaktiven $\mathrm{Si}-\mathrm{P}$-Bindung immer mehr zum wertvollen Ausgangsprodukt für Organophosphorverbindungen. So ist durch Umsetzung dieser Verbindung mit n-Butyllithium das reaktive $\left[\left(\mathrm{CH}_{3}\right)_{3} \mathrm{Si}\right]_{2} \mathrm{PLi} \cdot \ddot{\mathrm{Atther}}{ }^{1)}$ darstellbar. Die Reaktion mit Carbonsäure-halogeniden führt zu Acylphosphinen und ihren Derivaten ${ }^{2)}$, unter denen die Diacylphosphine wegen ihrer Keto-Enol-Tautomerie (zwischen $\mathrm{PH}$ und $\mathrm{O}-\mathrm{H}-\mathrm{O}$ ) besonders hervorzuheben sind ${ }^{3}$.

Tris(trimethylsilyl)phosphin wurde lange Zeit durch Umsetzung von $\mathrm{NaPH}_{2}{ }^{4)}$ (aus $\mathrm{PH}_{3}$ und $\mathrm{Na}$ in fl. $\mathrm{NH}_{3}$ ) oder $\mathrm{Li}_{3} \mathbf{P}^{5)}$ (aus $\mathbf{P H}_{3}$ und n-Butyllithium) mit Trimethylchlorsilan in Diglyme dargestellt. Bei allen Ansätzen muß mit größeren Mengen gasförmigen Phosphins gearbeitet werden. Zur kürzlich publizierten Methode von Schumann und Rösch ${ }^{6}$ (aus $\mathrm{Mg},\left(\mathrm{CH}_{3}\right)_{3} \mathrm{SiCl}$ und $\mathrm{PCl}_{3}$ in HMPTA bzw. THF) ist zu bemerken, daß die von den Autoren angegebenen Ausbeuten nicht immer erreicht werden, da offenbar die Reaktionsbedingungen nicht hinreichend bekannt sind.

Vor einiger Zeit berichteten Peterson und $\operatorname{Logan}^{7)}$ über die Reaktion von gelöstem weißem Phosphor in Benzol mit $\mathrm{Na} / \mathrm{K}$-Legierung zum, $\mathrm{Na}_{3} \mathrm{P} / \mathrm{K}_{3} \mathrm{P}$ “ und die anschließende Umsetzung mit Methylhalogeniden. Wir fanden, daß auf diese Weise dargestelltes „Na ${ }_{3} \mathrm{P} / \mathrm{K}_{3} \mathrm{P}$ “ (nach $\mathrm{Ab}$ pipettieren des überstehenden Benzols) in Monoglyme oder Diglyme in befriedigender Ausbeute mit Trimethylchlorsilan zum Tris(trimethylsilyl)phosphin reagiert. Allerdings kann auf diese Weise dargestełltes Tris(trimethylsilyl)phosphin bis zu 10\% 3,6-Bis(trimethylsilyl)-1,4-cyclohexadien enthalten, das durch Reduktion des Benzols und anschließende Silylierung entsteht ${ }^{8)}$ und auch durch Destillation über eine Ringspaltkolonne nicht abzutrennen ist. Führt man auch die Reduktion des weißen Phosphors in Monoglyme durch, so unterbleibt bei gleicher Ausbeute die Bildung des Nebenproduktes.

Wir danken Herrn Prof. Dr. G. Fritz für die Unterstützung dieser Arbeit, Herrn Dr. H. Scheer für die Aufnahme der Massen-, Herrn H. Domnick für die Aufnahme der ${ }^{1}$ H-NMR-Spektren. Die Bayer $A G$, Leverkusen, unterstützte uns mit Chemikalien.

1) G, Fritz und W. Hölderich, zur Veröffentlichung vorbereitet.

2) G. Becker, zur Veröffentlichung vorbereitet.

3) G. Becker und H.P.Beck, $2^{\text {nd }}$ IUPAC-Symposium on Inorganic Phosphorus Compounds, Prag, Sept. 1974, Bound of Abstracts 5.5, 112.

4) A. B. Bruker, L. D. Balashova und L. Z. Soborovskii, Dokl. Akad. Nauk SSSR 135, 843 (1960) [C. A. 55, 13301 a (1961)]; A. J. Leffler und E. G. Teach, J. Amer. Chem. Soc. 82, 2710 (1960).

5) G. W. Parshall und R. V. Lindsey jr., J. Amer. Chem. Soc. 81, 6273 (1959).

6) H. Schumann und L. Rösch, J. Organomet. Chem. 55, 257 (1973); Chem. Ber. 107, 854 (1974).

7) D. J. Peterson und T. J. Logan, J. Inorg. Nucl. Chem. 28, 53 (1966).

8) D. R. Weyenberg und L. H. Toporcer, J. Amer. Chem. Soc. 84, 2843 (1962); T. Brennan und H. Gilman, J. Organomet. Chem. 12, 291 (1968). 


\section{Experimenteller Teil}

Alle Arbeiten wurden unter getrocknetem Reinstargon ausgeführt.

Tris(trimethylsilyl)phosphin: $50.5 \mathrm{~g}$ (1.29 mol) Kalium und $39.3 \mathrm{~g}$ (1.71 mol) Natrium (schneiden, mit Benzol waschen, Benzolreste i. Vak. entfernen) werden in einem Kolben mit seitlich angesetzter G2-Fritte zusammengeschmolzen. Von Verunreinigungen wird abfiltriert. $31 \mathrm{~g}(1 \mathrm{~mol})$ getrockneter weißer Phosphor werden in einem 2-Liter-Dreihalskolben mit KPG-Rührer, Rückflußkühler und Tropftrichter unter Monoglyme ( $800 \mathrm{ml}$ ) geschmolzen. Dabei bilden sich zwei Phasen. Die flüssige $\mathrm{Na} / \mathrm{K}$-Legierung wird unter starkem Rühren direkt - ohne die Kolbenwand zu berühren - in die Suspension getropft. Dabei bildet sich anfänglich gelbliches, später schwarzes "Na ${ }_{3} \mathrm{P} / \mathrm{K}_{3} \mathrm{P}^{*}$. Zur Vervollständigung der Reaktion wird noch $24 \mathrm{~h}$ weiter erhitzt. Dann läßt man $325 \mathrm{~g}(3 \mathrm{~mol})$ Trimethylchlorsilan zutropfen und hält weitere $48 \mathrm{~h}$ am Sieden. Es wird filtriert und der Rückstand mit viel Lösungsmittel (2 Liter Monoglyme oder Benzol) gewaschen. Aus dem nach Abdestillieren des Lösungsmittels hinterbleibenden Rückstand lassen sich $163 \mathrm{~g}(0.65 \mathrm{~mol})$ reines Tris(trimethylsilyl)phosphin isolieren (Sdp. $46-50^{\circ} \mathrm{C} / 1 \cdot 10^{-3} \mathrm{Torr}$; Ausb. $60-75 \%$ ). 